BULL. AUSTRAL. MATH. SOC.

VOL. 28 (1983), 367-381.

\title{
PROLONGATIONS OF LINEAR CONNECTIONS TO THE FRAME BUNDLE
}

\author{
Luis A. Cordero and Manuel de Leon
}

\begin{abstract}
In this paper we construct the prolongation of a linear connection $\Gamma$ on a manifold $M$ to the bundle space $F M$ of its frame bundle, and show that such prolongated connection coincides with the so-called complete lift of $\Gamma$ to FM.
\end{abstract}

\section{Introduction}

The purpose of the present paper is to construct the prolongation of a linear connection on a manifold $M$ to the bundle space $\underline{\underline{F}} M$ of the frame bundle of $M$. To do this, we use Morimoto's general theory of prolongations to tangential fibre bundles of $p^{r}$-jets of $M$ [6] particularized when $r=1$, as well as some result stated in [2].

In $\S 1$, we briefly recall some results which will be used in the remaining sections. In $\S 2$, the prolongation of a connection on a principal fibre bundle $P$ to the principal bundle $J_{p}^{1} p$ of $p^{1}$-jets of $P$ is constructed. In $\S 3$, we apply the results in $\S 2$ for the case of linear connections and construct the prolongation $\tilde{\Gamma}$ to $F M$ of a linear connection $\Gamma$ on $M$, proving moreover that $\tilde{\Gamma}$ coincides with the socalled complete lift $\Gamma^{c}$ of $\Gamma$ defined by Mok in [5]. Finally, in $\$ 4$ we show that connections adapted to G-structures on $M$ prolongate to

Received 18 July 1983.

Copyright Clearance Centre, Inc. Serial-fee code: 0004-9727/83 $\$$ A2.00 +0.00 . 
connections adapted to the corresponding prolongations of these Gstructures introduced in [2].

In this paper all manifolds and mappings are assumed to be differentiable of class $c^{\infty}$, entries of matrices are written as $a_{j}^{i}, i$ being the row index and $j$ the column index, and summation over repeated index is always implied.

\section{Preliminaries}

Let $M$ be an $n$-dimensional manifold, $R^{p}$ the Euclidean $p$-space and $J_{p}^{1} M$ the set of 1 -jets at $0 \in R^{p}$ of all differentiable mappings $\delta: R^{p} \rightarrow M$ defined on some open neighborhood of $0 \in R^{p} ;$ if $j^{1}(6)$ denotes the 1 -jet of 6 at 0 , the target map $\pi: J_{p}^{1} M \rightarrow M$ is defined by $\pi\left(j^{\perp}(\delta)\right)=\delta(0)$ and is in fact a projection map from $J_{p}^{I} M$ onto $M$.

On $J_{p}^{l} M$ there exists a structure of $(n+p n)$-dimensional manifold, canonically induced from the manifold structure of $M$, which is given as follows: let $\left(u, x^{i}\right)$ be a coordinate system in $M, U$ being the coordinate neighborhood and $\left\{x^{i}\right\}$ the coordinate functions on $u$; then, on $J_{p}^{1} U=\pi^{-1}(U)$ we define a family of coordinate functions $\left\{x^{i}, x_{\alpha}^{i}\right\}$ by setting

$$
x^{i}\left(j^{1}(\delta)\right)=x^{i}(\sigma(0)), \quad x_{\alpha}^{i}\left(j^{1}(\sigma)\right)=\left.\frac{\partial\left(x^{i} \circ b\right)}{\partial t^{\alpha}}\right|_{0}
$$

$(1 \leq i \leq n, 1 \leq \alpha \leq p)$ for any $j^{1}(\sigma) \in j_{p}^{1} u$, and where $\left(t^{1}, \ldots, t^{p}\right)$ are the canonical coordinate functions on $R^{p}$. Then $\left(j_{p}^{1} u, x^{i}, x_{\alpha}^{i}\right)$ is a coordinate system in $J_{p}^{1} u$ which will be said to be induced by $\left(u, x^{i}\right)$ in $M$.

Let $h: M \rightarrow N$ be a differentiable map; then $h^{l}: J_{p}^{I} M \rightarrow J_{p}^{1} N$ will 
denote the map canonically induced by $h$ and given by $h^{l}\left(j^{l}(\sigma)\right)=j^{l}(h \circ \sigma)$ for any $j^{1}(\sigma) \in J_{p}^{1} M$. If $\left(u, x^{i}\right),\left(u^{\prime}, y^{j}\right)$ are local coordinate systems in $M$ and $N$ respectively, and if we assume $h: u \rightarrow u^{\prime}$ expressed by $y^{j}=h^{j}\left(x^{1}, \ldots, x^{n}\right)$ then, with respect to the induced coordinate systems $\left(J_{p}^{\perp} u, x^{i}, x_{\alpha}^{i}\right),\left(J_{p}^{I} u^{\prime}, y^{j}, y_{\alpha}^{j}\right), h^{\mathcal{L}}$ is expressed by

$$
h^{1}: y^{j}=h^{j}\left(x^{1}, \ldots, x^{n}\right), y_{\alpha}^{j}=\frac{\partial h^{j}}{\partial x^{k}} x_{\alpha}^{k},
$$

where $1 \leq k \leq \operatorname{dim} M, I \leq j \leq \operatorname{dim} N$ and $l \leq \alpha \leq p$.

Let $G$ be a Lie group; then $J_{p}^{l} G$ has also a Lie group structure, its product being defined as follows: for any $j^{\perp}(f), j^{\perp}(g) \in J^{\perp} G$, $j^{l}(f) \cdot j^{l}(g)=j^{l}(6 g)$, where $6 g: R^{p} \rightarrow G$ is defined by $(f g)(t)=f(t) g(t), t \in \operatorname{dom} 6 n$ dom $g$. The unit element $e_{p}$ of ${ }_{p}^{I} G$ is then the l-jet at $0 \in R^{p}$ of the constant map from $R^{p}$ into the unit element $e$ of $G$.

Next, we shall recall some results to be used later.

(1) Assume $p=n=\operatorname{dim} M$. Then the bundle space $\underline{\underline{F}}$ of the principal fibre bundle of linear frames over $M$ (briefly, the frame bundle of $M$ ) is an open (dense) submanifold of $J_{n}^{l} M$, and the induced structure on FM is the usual one with respect to which $\pi_{M}: \underline{=} M \rightarrow M$ is a $G I(n)-$ principal bundle, $G l(n)$ denoting the general linear group. If $\left(u, x^{i}\right)$ is a local coordinate system in $M$, the induced coordinate functions on $\underline{F} U=\left(\pi_{M}\right)^{-1}(u)$ will be written as $\left(x^{i}, x_{j}^{i}\right)$ if there is no confusion.

(2) Assume $p=1$. Then $\pi: J_{1}^{I} M \rightarrow M$ is nothing but the tangent bundle $\pi_{M}: T M \rightarrow M$. In this case, if $\left(U, x^{i}\right)$ is a local coordinate system in $M$, the induced coordinate functions on $T U=\left(\pi_{M}\right)^{-1}(U)$ will be 
written as $\left(x^{i} ; \dot{x}^{i}\right)$. Note that the linear structure of this vector bundle is locally given as follows: let $x, y$ be tangent vectors at $x=\left(x^{1}, \ldots, x^{n}\right) \in u$ with coordinates $x=\left(x^{i} ; \dot{x}^{i}\right), y=\left(x^{i} ; \dot{y}^{i}\right)$; then $x+y=\left(x^{i} ; \dot{x}^{i}+\dot{y}^{i}\right)$. If $f: M \rightarrow N$, we shall denote by $T_{6}: T M \rightarrow T N$ the induced map.

(3) Let $P(M, \pi, G)$ be a principal fibre bundle with bundle space $P$, base space $M$, projection $\pi$ and structure group $G$. Then $J_{p}^{\mathcal{I}} P\left(J_{p}^{\perp}, \pi^{I}, J_{p}^{l} G\right)$ is again a principal fibre bundle. In fact, if $\phi_{U}: \pi^{-1}(U) \rightarrow U \times G$ is the trivialization of $P$ over $U \subset M$, then, since $\left(\pi^{1}\right)^{-1}\left(J_{p}^{1} u\right)=J_{p}^{\perp} \pi^{-1}(u)$, we define $\tilde{\phi}_{U}: J_{p}^{I} \pi^{-1}(U) \rightarrow J_{p}^{I} u \times J_{p}^{I} G$ by setting $\tilde{\phi}_{u}\left(j^{l}(\sigma)\right)=\left(j^{1}(\pi \circ \sigma), j^{1}\left(n \circ \phi_{u} \circ \delta\right)\right)$ for any $j^{1}(\delta) \in J_{p}^{1} \pi^{-1}(u)$, where $\eta: U \times G \rightarrow G$ is the canonical projection.

(4) Let $G=\mathrm{Gl}(n),\left\{x_{j}^{i}\right\}$ be the canonical coordinates in $\mathrm{Gl}(n)$, $\left\{x_{j}^{i}, x_{j \alpha}^{i}\right\}$ the induced coordinates in $J_{n}^{\perp} G I(n)$ and $\left\{y_{B}^{A}, 1 \leq A, B \leq n+n^{2}\right\}$ the canonical coordinates in $\mathrm{GI}\left(n+n^{2}\right)$; then, there exists a canonical embedding of Lie groups

$$
j_{n}: J_{n}^{l} \mathrm{Gl}(n)+\mathrm{Gl}\left(n+n^{2}\right)
$$

given by

$$
j_{n}\left(\left(x_{j}^{i}, x_{j \alpha}^{i}\right)\right)=\left[\begin{array}{cccc}
\left(x_{j}^{i}\right) & 0 & \cdots & 0 \\
\left(x_{j 1}^{i}\right) & \left(x_{j}^{i}\right) & \cdots & 0 \\
\vdots & \vdots & \ddots & \vdots \\
\left(x_{j n}^{i}\right) & 0 & \cdots & \left(x_{j}^{i}\right)
\end{array}\right]
$$

that is, with respect to the coordinates above $j_{n}$ is expressed by 


$$
\begin{aligned}
y_{j}^{i} & =x_{j}^{i}, y_{j_{\alpha}}^{i}=0, \\
j_{n}: y_{j}^{i}=x_{j \alpha}^{i}, y_{j_{\beta}}^{i} & =\delta_{B}^{\alpha} x_{j}^{i},
\end{aligned}
$$

where $i_{\alpha}=\alpha n+i, 1 \leq i, \alpha \leq n$. If we consider the Lie algebras of $\mathrm{J}_{n}^{1} \mathrm{Gl}(n)$ and $\mathrm{Gl}\left(n+n^{2}\right)$ identified with the tangent spaces at the respective unit elements $e_{n}$ and $e$, then the induced homomorphism

$$
j_{n}: T_{e} J_{n}^{l} \mathrm{Gl}(n) \rightarrow T_{e} \mathrm{Gl}\left(n+n^{2}\right)
$$

may be written as follows:

$$
j_{n}\left(\left(\delta_{j}^{i}, 0 ; A_{j}^{i}, B_{j \alpha}^{i}\right)\right)=\left(\delta_{B}^{A} ;\left[\begin{array}{cccc}
\left(A_{j}^{i}\right) & 0 & \ldots & 0 \\
\left(B_{j 1}^{i}\right) & \left(A_{j}^{i}\right) & \cdots & 0 \\
\vdots & \vdots & \ddots & \vdots \\
\left(B_{j n}^{i}\right) & 0 & \cdots & \left(A_{j}^{i}\right)
\end{array}\right)\right) \text {. }
$$

(5) Let $F M\left(M, \pi_{M}, G(n)\right)$ be the frame bundle of $M$, $J_{n}^{I} \underline{\underline{F}} M\left(J_{n}^{I} M, \pi_{M}^{I}, J_{n}^{l} G l(n)\right)$ the induced $J_{n}^{I} G l(n)$-principal bundle and ${ }^{F} J_{n}^{I} M\left(J_{n}^{I} M, \pi{ }_{J_{n}^{I} M}, G I\left(n+n^{2}\right)\right)$ the frame bundle of the $\left(n+n^{2}\right)$-dimensional manifold $J_{n}^{\perp}$. Then there exists a canonical injective homomorphism of principal bundles [2]

$$
j_{M}: J_{n=}^{I} \rightarrow \underline{\underline{F}}_{n}^{I}{ }_{n}^{M}
$$

over the identity of $J_{n}^{1} M$, with associate Lie group homomorphism $j_{n}$. The homomorphism $j_{M}$ is locally defined as follows: let $\left(u, x^{i}\right)$ be a local coordinate system in $M$ and consider fibered coordinate functions $\left(x^{i}, x_{\alpha}^{i}, x_{j}^{i}, x_{j \alpha}^{i}\right)$ on $\left(\pi_{M}^{1}\right)^{-1}\left(J_{n}^{1} u\right)$ and $\left(y^{i}, y_{\alpha}^{i}, y_{B}^{A}\right)$ on ${ }_{=}^{I} J_{n}^{1} u$; then, with respect to these coordinates, $j_{M}$ is expressed by 


$$
\begin{aligned}
y^{i}=x^{i}, \quad y_{\alpha}^{i} & =x_{\alpha}^{i}, \\
j_{M}: y_{j}^{i} & =x_{j}^{i}, y_{j_{\alpha}}^{i}=0, \\
y_{j}^{i} & =x_{j \alpha}^{i}, y_{j_{\beta}}^{i}=\delta_{\beta}^{\alpha} x_{j}^{i} .
\end{aligned}
$$

Since the restriction $\left.\stackrel{F}{=} J_{n}^{I} M\right|_{\underline{\underline{F}} M}$ of $\stackrel{\underline{F}}{=}{ }_{n}^{I} M$ to the open submanifold $\underline{\underline{F}} M \subset J_{n}^{1} M$ is canonically isomorphic to the frame bundle $\underline{\underline{F F}}$ of $\underline{\underline{F M}}$, then the homomorphism $j_{M}$ above induces an injective homomorphism of principal bundles, noted again $j_{M}:\left.J_{n}^{1} \underline{\underline{F}}\right|_{\underline{\underline{F M}}} \rightarrow \underline{\underline{F F} M}$, over the identity of $\underline{\underline{F} M}$ and with associate Lie group homomorphism $j_{n}$.

(6) Particularizing the general results of Morimoto ([6], Chapter IV), we can assert: let $M$ be an $n$-dimensional manifold; then there exist canonical diffeomorphisms

$$
\alpha_{M}^{p, I}: T J_{p}^{I} M \rightarrow J_{p}^{I} T M, \quad \alpha_{M}^{1, p}: J_{p}^{\perp} T M \rightarrow T J_{p}^{1} M
$$

such that $\alpha_{M}^{p, I}$ and $\alpha_{M}^{l, p}$ are mutually inverse. Locally, $\alpha_{M}^{p, I}$ is given as follows: let $\left(u, x^{i}\right)$ be a local coordinate system in $M$ and let $\left(x^{i}, x_{\alpha}^{i} ; \dot{x}^{i}, \dot{x}_{\alpha}^{i}\right),\left(y^{i}, \dot{y}^{i},\left(y^{i}\right)_{\alpha},\left(\dot{y}^{i}\right)_{\alpha}\right)$ be the induced coordinate functions on $T J_{p}^{I} U$ and $J_{p}^{I} T U$ respectively. Then

$$
\alpha_{M}^{p, I}: y^{i}=x^{i}, \quad \dot{y}^{i}=\dot{x}^{i},\left(y^{i}\right)_{\alpha}=x_{\alpha}^{i},\left(\dot{y}^{i}\right)_{\alpha}=\dot{x}_{\alpha}^{i},
$$

with $1 \leq i \leq n, I \leq \alpha \leq p$. The local expression of $\alpha_{M}^{I, p}$ is obvious. Moreover, if $f: M \rightarrow N$ is a differentiable map, then the following diagram is commutative 


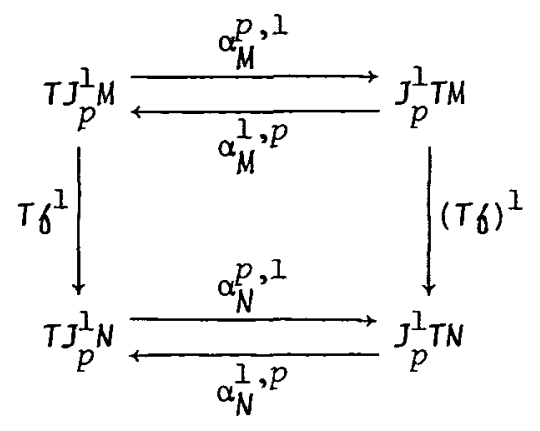

\section{Prolongation of connections}

Let $P(M, \pi, G)$ be a principal fibre bundle and consider on $P$ a connection whose connection form will be denoted by $\omega$. Following Kobayashi [3], we shall consider this form $\omega$ as a differentiable map $\omega: T P \rightarrow T G$ which is a linear map of the tangent space $T_{u} P$ with values in the tangent space $T_{e} G$ for each point $u \in P$, and satisfying:

$$
\begin{aligned}
& \omega(u \cdot \bar{s})=s^{-1} \cdot \bar{s}, \\
& \omega(\bar{u} \cdot s)=s^{-1} \cdot \omega(\bar{u}) \cdot s,
\end{aligned}
$$

for every $u \in P, s \in G, \bar{u} \in T_{u} P$ and $\bar{s} \in T_{s} G$, and where by definition $\bar{u} \cdot s=T R_{s}(\bar{u}), u \cdot \bar{s}=T L_{u}(\bar{s}), R_{s}: P \rightarrow P$ and $L_{u}: G \rightarrow P$ being the canonical maps.

Let $\omega: T P \rightarrow T G$ be a connection form on $P(M, \pi, G)$ and define a differentiable map $\omega_{1}: T J_{p}^{I} P \rightarrow T J_{p}^{I} G$ by setting

$$
\omega_{1}=\alpha_{G}^{1, p} \circ \omega^{1} \circ \alpha_{p}^{p, 1} \text {. }
$$

Then, from Morimoto's general results [6], we know that

$$
\begin{gathered}
\operatorname{Im} \omega_{1} \subset T_{e_{p}}^{J_{p}^{1} G,} \\
\omega_{1}(\tilde{u} \cdot \tilde{\bar{s}})=\tilde{s}^{-1} \cdot \tilde{\bar{s}}, \\
\omega_{1}(\tilde{\bar{u}} \cdot \tilde{s})=\tilde{s}^{-1} \cdot \omega_{1}(\tilde{\bar{u}}) \cdot \tilde{s},
\end{gathered}
$$


for every $\tilde{s} \in J_{p}^{I} G, \tilde{u} \in J_{p}^{I} p, \tilde{\bar{s}} \in \tau_{\tilde{s}^{\prime}}^{J_{p}^{I} G}$ and $\tilde{\bar{u}} \in T_{\tilde{u}} J_{p}^{I} p$. Hence, to prove that $\omega_{1}$ is actually a connection form on the principal bundle $J_{p}^{\perp} p\left(J_{p}^{\perp} M, \pi^{\perp}, J_{p}^{\perp} G\right)$ it suffices to prove that $\omega_{\perp}: T_{\tilde{u}} J_{p}^{\perp} p \rightarrow T_{e_{p}} J_{p}^{\perp} G$ is a linear map for any $\tilde{u} \in J_{p}^{l} p$.

To do this we proceed as follows.

Let $\left(u, x^{i}\right),\left(u^{\prime}, y^{a}\right)$ be local coordinate systems in $P$ and $G$, respectively, with $u=\pi(\tilde{u}) \in U, e \in U^{\prime}$ and $1 \leq i \leq \operatorname{dim} P$, $1 \leq a \leq \operatorname{dim} G$. Then, with respect to the induced coordinate systems $\left(T U, x^{i}, \dot{x}^{i}\right),\left(T U^{\prime}, y^{a}, \dot{y}^{a}\right)$ in TP and TG respectively, $\omega$ is expressed by

$$
\omega: y^{a}=\omega^{a}\left(x^{i} ; \dot{x}^{i}\right)=y^{a}(e), \quad \dot{y}^{a}=\dot{\omega}^{a}\left(x^{i} ; \dot{x}^{i}\right),
$$

and, therefore, for any $i$ and $a$,

$$
\frac{\partial \omega^{a}}{\partial x^{i}}=\frac{\partial \omega^{a}}{\partial \dot{x}^{i}}=0 .
$$

On the other hand, if $\bar{u}, \bar{u}^{\prime} \in T_{u} p$ are given by $\bar{u}=\left(x^{i} ; \dot{x}^{i}\right)$, $\bar{u}^{\prime}=\left(x^{i} ; \dot{x}, \dot{i}\right)$ then the linearity of $\omega: T_{u} P \rightarrow T_{e} G$ implies

$$
\dot{\omega}^{a}\left(x^{i} ; \dot{x}^{i}+\dot{x}, \dot{ }{ }^{i}\right)=\dot{\omega}^{a}\left(x^{i} ; \dot{x}^{i}\right)+\dot{\omega}^{a}\left(x^{i} ; \dot{x}^{\prime}\right)
$$

and therefore

$$
\begin{aligned}
& \frac{\partial \dot{\omega}^{a}}{\partial x^{i}}\left(x^{i} ; \dot{x}^{i}+\dot{x}, i\right)=\frac{\partial \dot{\omega}^{a}}{\partial x^{i}}\left(x^{i} ; \dot{x}^{i}\right)+\frac{\partial \dot{\omega}^{a}}{\partial x^{i}}\left(x^{i} ; \dot{x}, i\right), \\
& \frac{\partial \dot{\omega}^{a}}{\partial \dot{x}^{i}}\left(x^{i} ; \dot{x}^{i}+\dot{x}, i\right)=\frac{\partial \dot{\omega}^{a}}{\partial \dot{x}^{i}}\left(x^{i} ; \dot{x}^{i}\right)+\frac{\partial \dot{\omega}^{a}}{\partial \dot{x}^{i}}\left(x^{i} ; \dot{x}, i\right) .
\end{aligned}
$$

Now, let $\left(x^{i}, x_{\alpha}^{i} ; \dot{x}^{i}, \dot{x}_{\alpha}^{i}\right),\left(y^{a}, y_{\alpha}^{a} ; \dot{y}^{a}, \dot{y}_{\alpha}^{a}\right)$ be the induced coordinate functions on $T J_{p}^{l} U$ and $T J_{p}^{l} U^{\prime}$ respectively. Then, taking into account the local expressions of $\alpha_{p}^{p, I}, \alpha_{G}^{I, p}$ and $\omega^{\perp}$ as well as (2.2), a 
direct computation leads to the following local expression of $\omega_{1}$ :

$$
\begin{aligned}
y^{a} & =y^{a}(e), y_{\alpha}^{a}=0, \\
\omega_{1}: \dot{y}^{a} & =\dot{\omega}^{a}\left(x^{i} ; \dot{x}^{i}\right), \\
\dot{y}_{\alpha}^{a} & =\frac{\partial \dot{\omega}^{a}}{\partial x^{k}}\left(x^{i} ; \dot{x}^{i}\right) \cdot x_{\alpha}^{k}+\frac{\partial \dot{\omega}^{a}}{\partial \dot{x}^{k}}\left(x^{i} ; \dot{x}^{i}\right) \cdot \dot{x}_{\alpha}^{k} .
\end{aligned}
$$

Therefore, if $\tilde{u} \in J_{p}^{\mathcal{I}} U$ has coordinates $\tilde{u}=\left(x^{i}, x_{\alpha}^{i}\right)$ and $\tilde{\bar{u}}, \tilde{\bar{u}}{ }^{\prime} \in T_{\tilde{u}_{p}}^{J^{1}} u$ are given by $\tilde{\bar{u}}=\left(x^{i}, x_{\alpha}^{i} ; \dot{x}^{i}, \dot{x}_{\alpha}^{i}\right), \tilde{\bar{u}}^{\prime}=\left(x^{i}, x_{\alpha}^{i} ; \dot{x}^{\prime}, \dot{x}_{\alpha}, i\right)$ then $\tilde{\bar{u}}+\tilde{\bar{u}}^{\prime}=\left(x^{i}, x_{\alpha}^{i} ; \dot{x}^{i}+\dot{x}, i, \dot{x}_{\alpha}^{i}+\dot{x}_{\alpha}^{\prime i}\right)$ and a straightforward computation, using (2.3) and (2.4), leads to

$$
\begin{aligned}
& \dot{y}^{a}\left(\omega_{1}\left(\tilde{\bar{u}}+\tilde{\bar{u}}^{\prime}\right)\right)=\dot{y}^{a}\left(\omega_{1}(\tilde{\bar{u}})\right)+\dot{y}^{a}\left(\omega_{1}\left(\tilde{\bar{u}}^{\prime}\right)\right), \\
& \dot{y}_{\alpha}^{a}\left(\omega_{1}\left(\tilde{\bar{u}}+\overline{\bar{u}}^{\prime}\right)\right)=\dot{y}_{\alpha}^{a}\left(\omega_{1}(\tilde{\bar{u}})\right)+\dot{y}_{\alpha}^{a}\left(\omega_{1}\left(\tilde{\bar{u}}^{\prime}\right)\right) .
\end{aligned}
$$

Thus we have proved the following

THEOREM 2.1. Let $\omega: T P \rightarrow T G$ be a connection form on a principal fibre bundle $P(M, \pi, G)$. Then $\omega_{1}: T J_{p}^{1} P \rightarrow T J_{p}^{1} G$ given by (2.1) is a connection form on the principal fibre bundle $J_{p}^{1} p\left(J_{p}^{I} M, \pi^{I}, J_{p}^{I} G\right)$. We shall call $\omega_{1}$ the prolongation of the connection $\omega$ to $J_{p}^{I} p$.

We remark that, for $p=1, \omega_{1}$ coincides with the connection tangential to $\omega$ due to Kobayashi ([3], p. 152), also obtained by Morimoto in [7].

\section{Prolongation of linear connections to the frame bundle}

In this section we apply the result in the previous section to the linear connections on a manifold. From now on the indices $h, i, j, k, \ldots, \alpha, \beta, \gamma, \ldots$ have range in $\{1,2, \ldots, n\}$, $A, B, C, \ldots$ in $\left\{1,2, \ldots, n+n^{2}\right\}$ and $i_{\alpha}$ stands for $\alpha n+i$. 
Let $\underline{F} M\left(M, \pi_{M}, G l(n)\right)$ and $\underline{\underline{F F}} M\left(\underline{\underline{F}} M, \pi_{F M}, G l\left(n+n^{2}\right)\right)$ be the frame bundles of $M$ and $\underline{\underline{F}}$ respectively.

THEOREM 3.1. Let $\Gamma$ be a linear connection on a manifold $M$. Then there exists canonically a linear connection $\tilde{\Gamma}$ on the frame bundle $\underline{\underline{F}}$ of $M$, which will be called the prolongation of $\Gamma$ to $\underline{\underline{F}}$.

Proof. Let $\omega$ be the connection form on $\underline{\underline{F}}$ defining the connection $\Gamma$. The prolongation $\omega_{1}$ of $\omega$ is a connection form on $J_{n}^{\mathcal{F} M}$, $\dot{n}=\operatorname{dim} M$. Then, using the bundle homomorphism $j_{M}:\left.J_{n}^{\perp} \underline{\underline{F} M}\right|_{\underline{F} M} \rightarrow \underline{\underline{F F} M}$ described in $\$ 1,(5)$, we canonically obtain a connection $\tilde{\Gamma}$ on the principal fibre bundle $\underline{\underline{F F M}}$. \#

Next, we shall compute the local components $\tilde{\Gamma}_{B C}^{A}$ of the prolongation $\tilde{\Gamma}$ of $\Gamma$ to $\stackrel{F M}{=}$.

Let $\omega: T_{\underline{F} M} \rightarrow T_{G l}(n)$ be the connection form of $\Gamma,\left(u, x^{i}\right)$ a local coordinate system in $M,\left(x^{i}, x_{j}^{i}\right)$ the induced coordinate functions on $\underline{\underline{F}} U,\left(y_{j}^{i}\right)$ the canonical coordinates in $\mathrm{Gl}(n)$ and $\left(x^{i}, x_{j}^{i} ; \dot{x}^{i}, x_{j}^{i}\right)$, $\left(y_{j}^{i} ; \dot{y}_{j}^{i}\right)$ the induced coordinate functions on $T_{\underline{F}} U$ and $T_{G l}(n)$, respectively. Then $\omega$ is locally expressed by

$\omega$ :

$$
y_{j}^{i}=\omega_{j}^{i}\left(x^{h}, x_{k}^{h} ; \dot{x}^{h}, x_{k}^{h}\right)=\delta_{j}^{i},
$$$$
\dot{y}_{j}^{i}=\dot{\omega}_{j}^{i}\left(x^{h}, x_{k}^{h} ; \dot{x}^{h}, x_{k}^{h}\right) \text {, }
$$

and thus, if $\left\{e_{i}^{j}\right\}$ denotes the canonical basis of $g l(n) \equiv T_{e} G l(n)$, we can set

$$
\omega\left(x^{h}, x_{k}^{h} ; \dot{x}^{h}, x_{k}^{h}\right)=\dot{\omega}_{j}^{i}\left(x^{h}, x_{k}^{h} ; \dot{x}^{h}, x_{k}^{h}\right) e_{i}^{j} \in T_{e} G l(n) .
$$

Let $\sigma: U \rightarrow \underline{\underline{F} M}$ be the natural cross section of $\underline{\underline{F}}$ over $u$, that is $\sigma(x)=\left(x^{i}, \delta_{j}^{i}\right)$ for any $x=\left(x^{1}, \ldots, x^{n}\right) \in U$, and set $\omega_{u}=\sigma^{*} \omega$. 
Then $\omega_{U}$ defines the local components $\Gamma_{j k}^{i}$ of $\Gamma$ on $u$ by the equation $\omega_{u}=\left(r_{j k}^{i} d x^{j}\right) e_{i}^{k}$ and, using Proposition 7.3 in [4], one easily finds

$$
\dot{\omega}_{j}^{i}\left(x^{h}, x_{k}^{h} ; \dot{x}^{h}, \dot{x}_{k}^{h}\right)=y_{k}^{i} \Gamma_{h \tau^{k}}^{k} x_{j}^{2} \dot{x}^{h}+y_{h}^{i} \dot{x}_{j}^{h}
$$

where $\left(y_{k}^{i}\right)=\left(x_{k}^{i}\right)^{-1}$. Consequently, at the point $q=\left(x^{h}, x_{k}^{h} ; \dot{x}^{h}, x_{k}^{h}\right)$ we have

$$
\begin{aligned}
& \frac{\partial \dot{w}_{j}^{i}}{\partial x^{k}}(q)=v_{l}^{i}\left(\partial_{k} \Gamma_{h m}^{z}\right) x_{j}^{m \cdot x^{h}} \\
& \frac{\partial \dot{\omega}_{j}^{i}}{\partial x_{k}^{h}}(q)=-y_{h}^{i} x_{z}^{k} \Gamma_{m r}^{z} x_{j}^{r} x^{m}+y_{2}^{i} \Gamma_{m h}^{z} \dot{x}^{m} \delta_{k}^{j}-y_{h}^{i} x_{m}^{k} x_{j}^{m}, \\
& \frac{\partial \dot{\omega}_{j}^{i}}{\partial \dot{x}^{k}}(q)=y^{i} \Gamma_{k h j}^{2} \dot{x}_{j}^{h} \\
& \frac{\partial \dot{w}_{j}^{i}}{\partial k_{k}^{h}}(q)=y_{h}^{i} \delta_{k}^{j}
\end{aligned}
$$

Now, let $\tilde{\omega}$ denote the connection form of the extension of $\omega_{1}$ to

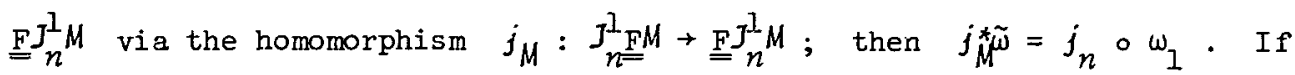

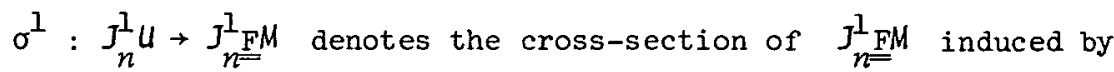
$\sigma: U \rightarrow F M$, then the composition $\tilde{\sigma}=j_{M} \circ \sigma^{1}$ is easily proved to be the natural cross-section of $\underline{F}_{n}^{I} M$ over $J_{n}^{I} u$.

Let $\left\{\tilde{\Gamma}_{B C}^{A}\right\}$ still denote the local components of the linear connection on $J_{n}^{l} M$ which is defined by $\tilde{\omega}$, with respect to the induced coordinate system $\left(J_{n}^{I} u, x^{j}, x_{\alpha}^{j}\right)$. Then, if $\left\{E_{B}^{A}\right\}$ denotes the canonical basis of gl $\left(n+n^{2}\right) \equiv T_{e} \mathrm{Gl}\left(n+n^{2}\right)$, we have 


$$
\tilde{\omega}\left(\left(\frac{\partial}{\partial x^{j}}\right)_{\tilde{u}}\right)=\tilde{\Gamma}_{j B}^{A} E_{A}^{B}, \quad \tilde{\omega}\left(\left(\frac{\partial}{\partial x_{\alpha}^{j}}\right)_{\tilde{u}}\right)=\tilde{\Gamma}_{j_{\alpha} B^{B} A} E^{B},
$$

$\tilde{u}=\tilde{\sigma}(u)$ for any point $u \in J_{n}^{\perp} u$. On the other hand, setting $u_{1}=\sigma^{1}(u)$

$$
\left(T_{j_{M}}\right)\left(\left(\frac{\partial}{\partial x^{j}}\right)_{u_{I}}\right)=\left(\frac{\partial}{\partial x^{j}}\right)_{\tilde{u}}, \quad\left(T_{j_{M}}\right)\left(\left(\frac{\partial}{\partial x_{\alpha}^{j}}\right)_{u_{1}}\right)=\left(\frac{\partial}{\partial x_{\alpha}^{j}}\right)_{\tilde{u}},
$$

and hence

$$
\begin{aligned}
& \tilde{\omega}\left(\left(\frac{\partial}{\partial x^{j}}\right)_{\tilde{u}}\right)=j_{n}\left(\omega_{1}\left(\left(\frac{\partial}{\partial x^{j}}\right)_{u_{1}}\right),\right. \\
& \tilde{\omega}\left(\left(\frac{\partial}{\partial x_{\alpha}^{j}}\right)_{\tilde{u}}\right)=j_{n}\left(\omega_{1}\left(\left(\frac{\partial}{\partial x_{\alpha}^{j}}\right)_{u_{1}}\right)\right) .
\end{aligned}
$$

Then, if $u=\left(x^{j}, x_{\alpha}^{j}\right)$, we have

$$
\begin{aligned}
\omega_{1}\left(\left(\frac{\partial}{\partial x^{j}}\right)_{u_{1}}\right) & =\omega_{1}\left(x^{i}, I, x_{\alpha}^{i}, 0 ; \delta_{j}^{i}, 0,0,0\right) \\
& =\left(I, 0 ; \dot{\omega}_{i}^{h}\left(x^{i}, I ; \delta_{j}^{i}, 0\right), \frac{\partial \omega_{i}^{h}}{\partial x^{k}} x_{\alpha}^{k}\right) \\
& =\left(I, 0 ; \Gamma_{j i}^{h}, x_{\alpha}^{k}\left(\partial_{k} \Gamma_{j i}^{h}\right)\right), \\
\omega_{1}\left(\left(\frac{\partial}{\partial x_{\gamma}^{j}}\right)_{u_{1}}\right) & =\omega_{1}\left(x^{i}, I, x_{\alpha}^{i}, 0 ; 0,0, \delta_{\gamma}^{\alpha} \delta_{j}^{i}, 0\right) \\
& =\left(I, 0 ; \dot{\omega}_{i}^{h}\left(x^{i}, I ; 0,0\right), \frac{\partial_{i}^{h}}{\partial \dot{x}^{k}} \delta_{j}^{k} \delta_{\gamma}^{\alpha}\right) \\
& =\left(I, 0 ; 0, \delta_{\gamma}^{\alpha}{ }_{j i}^{h}\right),
\end{aligned}
$$

where $I$ and 0 denote the unit matrix and the zero matrix, respectively. Therefore 


$$
\begin{aligned}
& \tilde{\omega}\left(\left(\frac{\partial}{\partial x^{j}}\right)_{\tilde{u}}\right)=\Gamma_{j i}^{h} E_{h}^{i}+x_{\alpha}^{k}\left(\partial_{k} \Gamma_{j i}^{h}\right) E_{h_{\alpha}}^{i}+\delta_{\beta}^{\alpha} \Gamma_{j i}^{h} E_{h_{\alpha}}^{i}, \\
& \tilde{\omega}\left(\left(\frac{\partial}{\partial x_{\gamma}^{j}}\right)_{\tilde{u}}\right)=\delta_{\gamma}^{\alpha} \Gamma_{j i}^{h} E_{h \alpha}^{i},
\end{aligned}
$$

and, restricting to $\underline{\underline{F}}$, that is to the coordinate neighborhood $\underline{\underline{F}}$, we obtain the local components of the prolongation $\tilde{\Gamma}$ of $\Gamma$ to $\underline{\underline{F}}$ :

$$
\begin{aligned}
& \tilde{\Gamma}_{j i}^{h}=\Gamma_{j i}^{h}, \tilde{\Gamma}_{j i_{\beta}}^{h}=0, \quad \tilde{\Gamma}_{j \gamma}^{h}=0, \tilde{\Gamma}_{j_{\gamma} i_{\beta}}^{h}=0, \tilde{\Gamma}_{j_{\beta} i_{\gamma}}^{h}=0, \\
& \tilde{\Gamma}_{j i}^{h}=x_{\alpha}^{k}\left(\partial_{k} \Gamma_{j i}^{h}\right), \quad \tilde{\Gamma}_{j i_{\beta}}^{h}=\delta_{\beta}^{\alpha} \Gamma_{j i}^{h}, \tilde{\Gamma}_{j_{\beta}{ }^{2}}^{h}=\delta_{\beta}^{\alpha} \Gamma_{j i}^{h} .
\end{aligned}
$$

Now, comparing with Mok's result in ([5], p. 81), we deduce

THEOREM 3.2. Let $\Gamma$ be a linear connection on $M$. Then the prolongation $\tilde{\Gamma}$ of $\Gamma$ to the frame bundle $\underline{F} M$ of $M$ coincides with the complete lift $^{C}$ of $\Gamma$ to $\underline{\underline{F} M}$ defined by Mok [5].

\section{Prolongation of connections adapted to G-structures}

We begin this section proving a lemma.

LEMMA 4.1. Let $P(M, \pi, G)$ be a reduced bundle of the principal fibre bundle $P^{\prime}\left(M, \pi, G^{\prime}\right)$, and let $\omega^{\prime}$ be a connection form on $P^{\prime}$ reducible to the connection form $\omega$ on $P$. Then $J_{p}^{1} p\left(J_{p}^{1} M, \pi^{1}, J_{p}^{1} G\right)$ is a reduced bundle of $J_{p}^{1} p^{\prime}\left(J_{p}^{1} M, \pi^{I}, J_{p}^{1} G^{\prime}\right)$, and the prolongation $\omega_{1}^{\prime}$ of $\omega^{\prime}$ to $J_{p}^{1} p^{\prime}$ is reducible to the prolongation $\omega_{1}$ of $\omega$ to $J_{p}^{1} p$.

Proof. Let $6: P \rightarrow P^{\prime}$ be the injective homomorphism of principal bundles which yields the reduction of $G^{\prime}$ to $G$, and denote also by $6: G+G^{\prime}$ the corresponding Lie group homomorphism. Then a straightforward computation shows that the induced bundle homomorphism $f^{l}: J_{p}^{1} p \rightarrow J_{p}^{1} p^{\prime}$ yields a reduction of $J_{p}^{1} G^{\prime}$ to $J_{p}^{1} G$ whose associate lie group homomorphism is the induced one, $\sigma^{1}: J_{p}^{1} G \rightarrow J_{p}^{1} G^{\prime}$. On the other 
hand, that $\omega^{\prime}$ is reducible to $\omega$ means that the following diagram commutes:

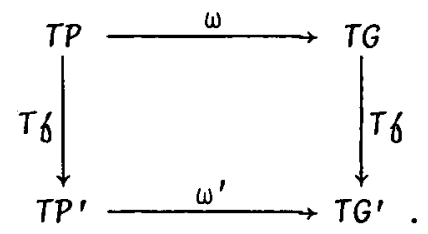

Therefore, from $(6)$ in $\S 1$ we obtain a new commutative diagram

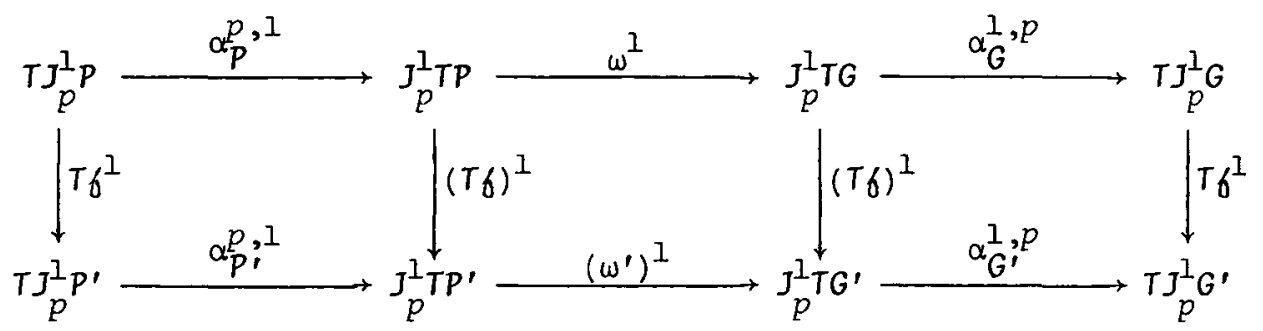

which implies that $\omega_{1}^{\prime}$ is reducible to $\omega_{1}$. \#

Let $G$ be a Lie subgroup of $G l(n)$ and denote $\tilde{G}=j_{n}\left(J_{n}^{1} G\right) \subset G I\left(n+n^{2}\right)$. Assume that $P(M, \pi, G)$ is a reduced bundle of the frame bundle $\underline{F} M\left(M, \pi_{M}, \mathrm{GI}(n)\right)$ of $M, n=\operatorname{dim} M$, that is $P$ is a G-structure on $M$. In [2] we have defined the prolongation of the G-structure $P$ on $M$ to a $\tilde{G}$-structure $\tilde{P}$ on $\underline{\underline{F}}$ as follows: we consider the injective bundle homomorphism $i^{\perp}: J_{n}^{\perp} p \rightarrow J_{n}^{I} \underline{\underline{F}}$ induced by $i: P \rightarrow \underline{\underline{F}} M$ and define $\tilde{P}=\left.\left(j_{M} \circ i^{l}\right)\left(j_{n}^{I} p\right)\right|_{\underline{\underline{F M}}}$.

As usually, we say that a linear connection $\Gamma$ on $M$ is adapted to the G-structure $P(M, \pi, G)$ on $M$ if $\Gamma$ is reducible to a connection on $P$. Then, taking into account Lemma 4.1 and the results in the previous section, we easily deduce

THEOREM 4.2. Let $\Gamma$ be a linear connection on $M$ adapted to a G-structure $P(M, \pi, G)$ on $M$. Then the prolongation $\tilde{\Gamma}$ of $\Gamma$ to $F$ is adapted to the $\tilde{G}$-structure $\tilde{P}(\underline{F} M, \pi, \tilde{G})$ on $F M$, prolongation of $\bar{P}$

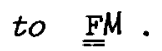

We remark that Theorem 4.2 improves some particular results in [1] and 
[5] where only the prolongations (or complete lift) of G-structures on $M$ defined by tensor fields of types $(0, s)$ and $(1, s)$ have been considered.

\section{References}

[1] Luis A. Cordero and Manuel de Leon, "Lifts of tensor fields to the frame bundle", Rend. Cir. Mat. PaZermo (to appear).

[2] Luis A. Cordero and Manuel de Leon, "Prolongations of G-structures to the frame bundle", submitted.

[3] S. Kobayashi, "Theory of connections", Ann. Mat. Pura App 2. 43 (1957), 119-194.

[4] S. Kobayashi and K. Nomizu, Foundations of differential geometry, Vol. I (Interscience, New York, 1963).

[5] K.P. Mok, "Complete lifts of tensor fields and connections to the frame bundle", Proc. London Math. Soc. (2) 32 (1979), 72-88.

[6] A. Morimoto, "Prolongation of geometric structures" (Mathematical Institut, Nagoya University, Japan, 1969).

[7] A. Morimoto, "Prolongation of connections to tangential fibre bundles of higher order", Nagoya Math. J. 40 (1970), 85-97.

Departamento de Geometria y Topologia,

Facultad de Matematicas,

Universidad de Santiago de Compostela, Spain. 